\title{
Multiple Fragmentation of Atoms in Femtosecond Laser Pulses
}

\author{
A. Becker ${ }^{1 *}$, R. Dörner ${ }^{2 \dagger}$, and R. Moshammer ${ }^{3} \ddagger$ \\ ${ }^{1}$ Max-Planck-Institut für Physik komplexer Systeme, \\ Nöthnitzer Str. 38, 01187 Dresden, Germany \\ ${ }^{2}$ Institut für Kernphysik, Max-von-Laue Str. 1, 60438 Frankfurt, Germany \\ ${ }^{3}$ Max-Planck-Institut für Kernphysik, \\ Saupfercheckweg 1, 69117 Heidelberg, Germany
}

(Dated: December 17, 2004)

\begin{abstract}
Multiple ionisation of atoms by an ultrashort intense laser pulse is a process in which the fewbody problem is closely interrelated with the highly nonlinear interaction between the electrons and the external field. We review recent advances in unveiling the mechanisms behind the unusually large ion yields for double and multiple ionisation observed in a strong laser pulse. Its study requires on the one hand the combination of highly differential experimental techniques with laser systems having high repetition rates and on the other the development of new theoretical methods to simultaneously account for the long-ranged Coulomb interaction between the particles and the field nonlinearity. Different mechanisms are analysed diagrammatically and quantitatively in comparison with experimental data for the total ion yields. Distributions for the electron and ion momenta of coincidence measurements are discussed along with predictions of the various theoretical methods.
\end{abstract}

\footnotetext{
* E-mail: abecker@mpipks-dresden.mpg.de

$\dagger$ E-mail: doerner@atom.uni-frankfurt.de

‡ E-mail: moshamme@mpi-hd.mpg.de
} 


\section{INTRODUCTION}

About 25 years after the quantum mechanical description of the photo-electric effect by Einstein [1] it was Göppert-Mayer [2] who predicted that the energies of more than one photon can be combined to achieve the emission of an electron from an atom or molecule, when the energy of one photon is not sufficient to overcome the binding potential. The observation of such multiphoton phenomena requires a high photon density at the location of the target, which became available with the evolution of the laser in the 1960s. Modern

laser systems (e.g. the Ti:sapphire lasers) provide peak light intensities of the order of $10^{20}$ $\mathrm{W} / \mathrm{cm}^{2}$ or above in pulses shorter than $100 \mathrm{fs}$. The field strength at these intensities is a hundred times the Coulomb field binding the ground state electron in the hydrogen atom [3]. These extreme photon densities allow highly nonlinear multiphoton processes such as abovethreshold ionisation, high harmonic generation, laser-induced tunneling, Coulomb explosion, multiple ionisation and others, where up to a few hundred photons can be absorbed from the laser field.

Single ionisation and other single-active-electron phenomena in intense fields have been intensively studied for many years. The experimental observables are the ionisation yields as a function of the laser intensity, the energy and angular distribution of the emitted electron or the emission of higher harmonic light. We refer the interested reader to several review articles covering this broad field [4-11].

In this review we focus on recent advances in unveiling the mechanisms of double and multiple ionisation in strong laser fields. It reveals new aspects of the electron-electron correlation, different from those in the ground state of atoms or in the double ionisation by charged particle (see [12] for a review) or single photon (see [13, 14] for reviews) impact. This few-body problem is not only an intellectual challenge, but it has also a wide ranging impact to many fields of science and technology. It is the correlated motion of electrons, which is responsible for the structure and the evolution of large parts of our macroscopic world. It drives chemical reactions and is the ultimate reason for superconductivity and many other effects in the condensed phase. In atomic processes few-body correlation effects can be studied in a particular clear manner.

With the increase of the electron number in the process the challenges in experimental observations and theoretical analysis increase. In the early experiments the yields of the 
multiply charged ions were measured. Recently it became possible to detect also the energy or the momentum vector of the electrons and the ions in coincidence by combining highly differential experimental techniques, that are standard in the fields of ion-atom, electron-atom and high energy single photon-atom collision studies, with laser systems having a kilohertz repetition rate. On the other hand, for an exact ab-initio simulation of a system with two electrons one needs to solve a set of six dimensional partial differential equations over a large space-time grid. Considerable progress has been made recently in this direction using high power computers [15-20]. An alternative ab-initio approach is provided by the IntenseField Many-Body $S$-Matrix Theory (IMST). It is a systematic approximation method for the analysis of processes which require to account simultaneously for different interactions of similar strength (in the present case the Coulombic interaction and the laser interaction). Further, a number of approximative methods have been developed, e.g. lower-dimensional numerical simulations or Classical Trajectory Monte Carlo calculations. Below we present and discuss recent observations and their analysis as well as the resulting physical picture of the correlated electron emission from an atom interacting with an intense laser pulse.

\section{MECHANISMS OF DOUBLE IONISATION IN STRONG FIELDS}

\section{A. Ionisation yields}

Single ionisation of atoms and molecules in intense laser pulses can be satisfactorily described using the single-active-electron (SAE) approximation [21], where only the outermost electron, moving in the effective potential of the ionic core and the other electrons, is assumed to interact with the field. In this picture the electron-electron interaction is negligible. It would imply for the double ionisation process that the electrons are emitted sequentially, i.e. the neutral atom gets first ionised, then the cation is further ionised independently of the first step et cetera. But, theoretical predictions based on this sequential ionisation mechanism do not account for the large double and multiple ionisation yields in intense laser fields observed at near infrared wavelengths. This is exemplified in Fig. 1 in plots of the ionisation yields as a function of the laser intensity. Here both double ionisation yields of He as observed by Walker et al. (Fig. 1(a), [22]) and multiple ionisation yields of Xe as measured by Larochelle et al. (1(c), [24]) are shown. At low intensities the experimental data 

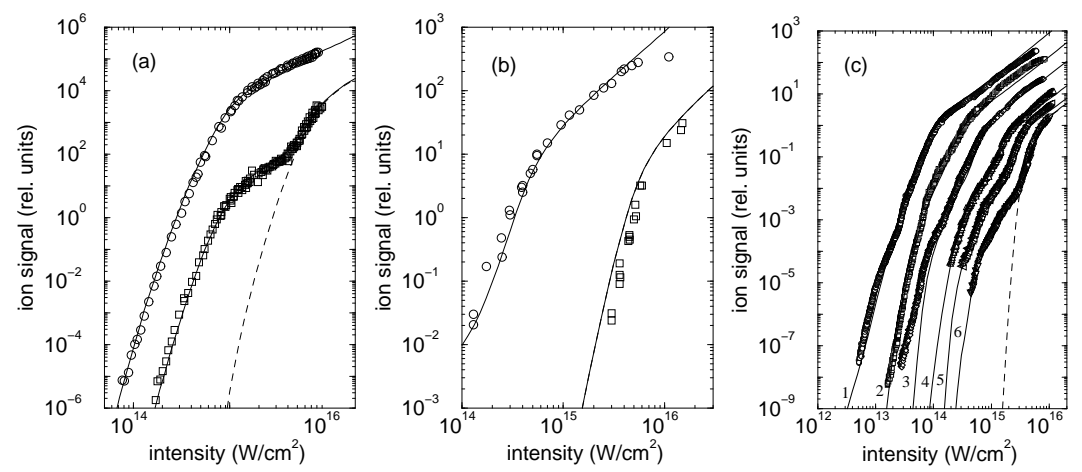

FIG. 1: Measured yields of double ionisation of Helium at $780 \mathrm{~nm}$ (panel a, [22]) and $248 \mathrm{~nm}$ (panel b, [23]), and multiple ionisation of Xenon at $800 \mathrm{~nm}$ (panel c, [24]) for linearly polarised laser light. Predictions of the $S$-matrix theory $[25,26]$ are shown as solid lines. Note the strong enhancement of the double and multiple ionisation yields over the sequential prediction at the infrared wavelengths (dashed lines) and its absence at $248 \mathrm{~nm}$.

show a strong enhancement of the double and multiple ionisation yields over the predictions

of the sequential mechanism (dashed lines, $S$-matrix calculations [25, 26], or nearly equal results of SAE simulations, see e.g. [22]). There is also the ubiquitous "knee"-structure to be seen in the double (and multiple) ionisation curves, which has been observed for the first time in the 1980s by L'Huillier et al. [27]. Note also that at $248 \mathrm{~nm}$ (Fig. 1(b)) no excessive double ionisation yield over the prediction of the sequential mechanism has been observed [23]. Thus, while at near infrared wavelengths a nonsequential ejection of two or more electrons dominates, there is no sign of it at the shorter UV wavelength.

\section{B. Mechanisms and diagrams}

What is the mechanism leading to nonsequential double (and multiple) ionisation? This seemingly well defined question does not necessarily have a unambiguous quantum mechanical answer, since it is not always straightforward to deduce such a picture from a theoretical analysis and even if this is possible often the contributions from different mechanisms have to be added coherently. An example is the single-photon double ionisation process at low light intensities. Here at least contributions of two mechanisms, called shake-off and twostep-one (their strong field analogous will be discussed below), have to be considered to 


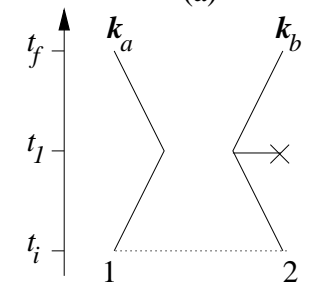

(b)

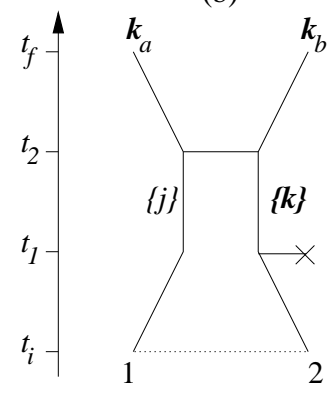

(c)

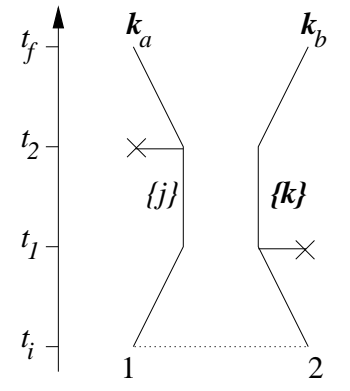

FIG. 2: Three (out of eight) diagrams that are generated by the the first leading terms of IMST. The diagrams correspond to qualitative mechanisms for nonsequential double ionisation proposed in the literature, namely (a) Shake-off, (b) Rescattering and (c) Sequential ionisation and Collective Tunneling.

obtain an agreement with the observations [28, 29]. This is not the case for nonsequential double ionisation in strong fields. As it will be seen below, there is one mechanism that dominates strongly.

Becker and Faisal have identified [30-32] diagrammatically and quantitatively the main mechanism of double ionisation using the Intense-Field Many-Body S-Matrix Theory (IMST). This theory is a thorough rearrangement of the usual $S$-matrix series such that all features of the process appear in the first leading terms of the series (for a review of IMST see [33]). In the case of double ionisation of an atom the IMST generates eight Feynman-like diagrams up to the second order of the series. Three of these diagrams are shown in Fig. 2, which is a form of representation of the $S$-matrix terms, that is convenient to obtain intuitive pictures or mechanisms of the process of interest. In the diagrams time is assumed to flow from the bottom upward and straight lines stand for the evolution of the two electrons. The three diagrams in Fig. 2 correspond to the following mechanisms qualitatively proposed before in the literature:

Shake-Off: If one electron is removed rapidly from an atom or a molecule by the in- 
teraction with an intense field, for example, the wave function of the remaining electron has to relax to the new eigenstates of the altered potential. Some of these states are in the continuum, so that a second electron can be shaken off during this relaxation process. This process corresponds to the first-order diagram in Fig. 2a. Here, the two electrons are initially (time $t_{i}$ ) in the ground state of the He atom. Electron correlation is included as indicated by the dotted line. At time $t_{1}$ one of the two electron leaves after the interaction with the field (denoted by ' $-\mathrm{x}$ ') so quickly that the second is shaken-off from the atom. The electrons propagate in the final state with momenta $\boldsymbol{k}_{a}$ and $\boldsymbol{k}_{b}$ in the presence of the field. The shake-off mechanism is well known for example from beta decay, where the nuclear charge is changed. It is also known to be one of the mechanisms for double ionisation by absorption or Compton scattering of a single photon (see the discussion in [34] and references therein). Its strong-field analog has been originally proposed by Fittinghoff et al. [35].

Rescattering: In diagram 2b one electron becomes active first and absorbs energy from the field at time $t_{1}$, at a later time $t_{2}$ it interacts with the second electron via the electronelectron interaction and the two electrons may emerge together from the atom. During the time interval $t_{2}-t_{1}$ the two electrons are in virtual intermediate states, namely one electron in the Volkov states (field dressed plane wave states) of momenta $\{\boldsymbol{k}\}$ and the second electron in intermediate states of the ion $\{j\}$. The diagram includes the rather analogous antenna picture, advanced by Kuchiev [36], and the semi-classical rescattering mechanism proposed by Corkum [37] and Schafer et al. [38]. According to the rescattering picture, first one electron is set free by quasi-static tunneling. Then it is accelerated by the laser field, gains energy and, depending on the phase of the field upon the moment of ejection, the electron can be driven back to its parent ion when the field changes its sign. Upon recollision with the ion the electron can either recombine and emit higher harmonic radiation, scatter elastically and get further accelerated or it scatters inelastically with simultaneous excitation or ionisation of the ion. Rescattering can be seen as a strong-field extension of the Two-StepOne mechanism (TS1), known for single photon double ionisation at low photon energies [39]. In the TS1 mechanism one electron absorbs the photon and knocks out the second one via an electron-electron collision on its way through the atom. Thus, while for the TS1 mechanism the electron correlation appears to be dominant on a very short time scale (a few attoseconds) and confined to a small region of space (the size of the electron cloud), in the rescattering mechanism there is a femtosecond time delay between the first and the 
second step. The diagram in Fig. 2(b) involves in the intermediate state both, short-time propagation $\left(t_{2}-t_{1}<\pi / 2 \omega\right.$, electron-electron collision on the way through the atom, TS1) and long time propagation $\left(t_{2}-t_{1}<\pi / 2 \omega\right.$, rescattering).

Collective tunneling: According to this mechanism the two electrons tunnel out simultaneously from the atom or molecule. It was advanced by Eichmann et al. [40] inspired by the fact that single ionisation at sufficiently high field strengths is well described by an one-electron tunneling formula (e.g. [41]). This mechanism is part of the $S$-matrix diagram in Fig. 2c. Here, the two electrons interact independently, at times $t_{1}$ and $t_{2}$ respectively, with the field and leave the atom. The diagram includes the sequential double ionisation for long intermediate times $t_{2}-t_{1}$ as well as a collective double ionisation for short intermediate times.

The experimental observation that double ejection is strongly suppressed in ionisation with circularly polarised light [42, 43] (see also figure 19 in [5]) provided strong evidence that the rescattering mechanism is dominantly responsible for double ionisation by strong laser fields. The rescattering mechanism is inhibited by the circular polarisation since the rotating electric field does not drive the electrons back to their parent ion. The other mechanisms, in contrast, are expected to be polarisation independent.

Becker and Faisal have also identified $[31,32]$ in their IMST analysis the rescattering diagram (Fig. 2b) as the dominant diagram for nonsequential double ionisation. For example, its contribution to the total ionisation rate was found to exceed by many orders of magnitude over that from the shake-off diagram [32]. It has been further shown by Zon [44] and Eichmann et al. [40] that the rate of collective two-electron tunneling is much too low to account for the large nonsequential double ionisation yields. The strong dominance of the rescattering mechanism for the ejection of two electrons also suggests [24, 26] a physical mechanism for nonsequential multiple ionisation, in which the first step of the process remains the same but the second is a generalisation to a $\left(\mathrm{e}^{-}, n \mathrm{e}^{-}\right)$collisional ionisation.

For the analysis of the ion yields of double and multiple ionisation Becker and Faisal proposed $[25,26,45,46]$ a simple model formula, which combines rates for single ionisation at the first stage of the process with collisional ionisation rates $\left(\left(\mathrm{e}^{-}, n \mathrm{e}^{-}\right), n=2,3, \ldots\right)$ at the second stage. Predictions of this model are found to be in good agreement with data of a large number of experiments $[25,26,47]$. As examples, the comparisons for the much discussed knee structure in double ionisation of He at near infrared wavelengths and for the 
case of up to six-fold ionisation of Xe as well as its absence at the UV wavelength are shown in Fig. 1 (solid lines). Before leaving this discussion of the total ion yields we note that it also has been shown experimentally and theoretically [46] that electrons from an inner shell can be ejected through the mechanism of nonsequential double and multiple ionisation.

\section{ANALYSIS OF COINCIDENCE MEASUREMENTS}

To gain further insight in the double ionisation process differential measurements which go beyond the measured total ion yields are necessary. For a long time, however, the experimental study of electron correlation has suffered from the technical challenge to observe more than one electron emerging from a multiple ionisation event. Only recently a breakthrough has been made by two types of such experiments: Electron time-of-flight measurements in coincidence with the ion charge state $[48-50,52]$ and those using the COLTRIMS (Cold Target Recoil Ion Momentum Spectroscopy, [53]) technique, where the ion momentum alone [54-60] or in coincidence with the momentum of one of the electrons [61-68] have been measured.

\section{A. Recoil ion momenta}

Recoil ion momentum distributions have been measured for helium $\left(\mathrm{He}^{+}, \mathrm{He}^{2+}\right)$ [54], neon $\left(\mathrm{Ne}^{+}-\mathrm{Ne}^{4+}\right)[55,60]$ and $\operatorname{argon}\left(\mathrm{Ar}^{1+}-\mathrm{Ar}^{4+}\right)[56,60,63]$. Fig. 3 summaries some of the experimental data for helium (upper row, [54]) in comparison with $S$-matrix results (lower row, [69]) for $800 \mathrm{~nm}$ laser light at $6.6 \times 10^{14} \mathrm{~W} / \mathrm{cm}^{2}$. Shown are the momentum components parallel (left hand panels) and perpendicular (right hand panels) to the field direction. The features of the distributions observed in the experiments are a distinct double peak structure for the parallel momentum component (Fig. 3a) and a single peak structure for the perpendicular component (Fig. 3b).

It has been shown $[54,55]$ that the double peak structure automatically rules out the shake-off [35] and the collective tunneling [40] mechanisms, since for both a strong peak at the origin would be expected. At the same time, the peaks were found to be in qualitative agreement with the recollision [37] or the antenna [36] mechanism. As mentioned above, in the rescattering picture there is a significant time delay between the emission of the first 

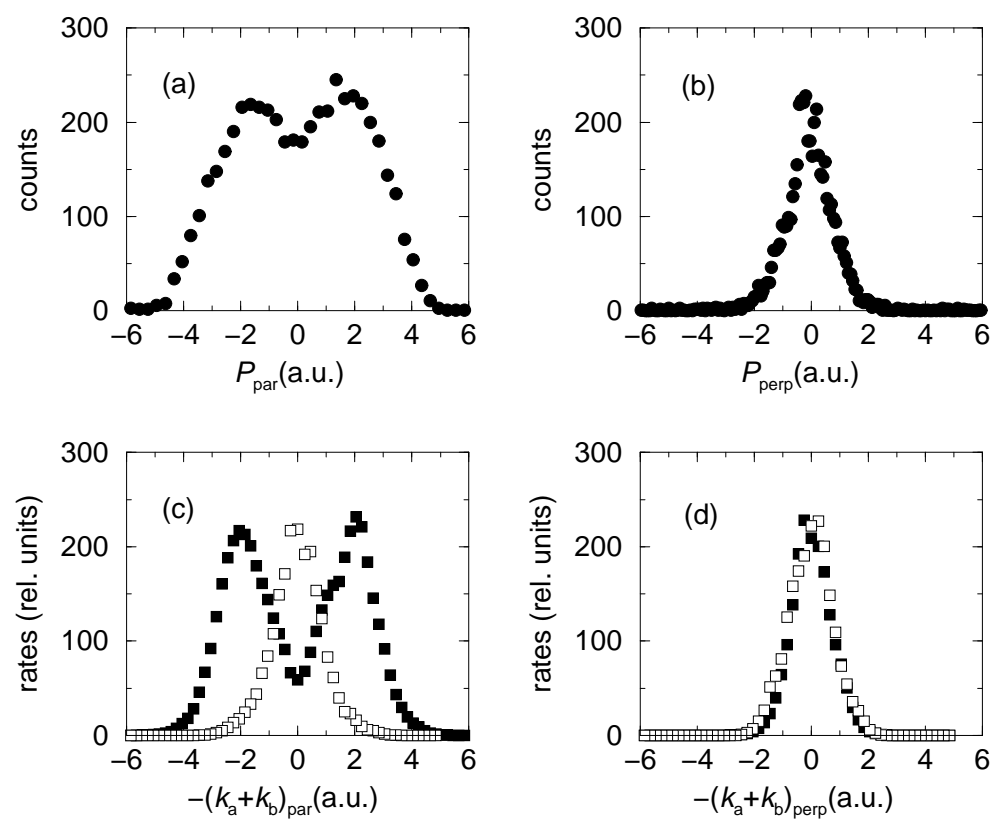

FIG. 3: Distributions of the recoil momentum of doubly charged Helium ions generated by 800 $\mathrm{nm}, 200 \mathrm{fs}$ laser pulses at $6.6 \times 10^{14} \mathrm{~W} / \mathrm{cm}^{2}$. Left hand panels: Components parallel to the field direction, right hand panels: Components perpendicular to the field direction. Experimental data (upper row, [54]) are compared with results of the $S$-matrix calculations [69] with (filled squares) and without (open squares) the final state Volkov dressing.

electron and the return to its parent ion. Estimating $t_{2}-t_{1}$ for a rescattering trajectory which has sufficient energy to ionise the residual ion leads to ion momenta close to the measured peak positions $[54,55,57]$.

Soon after the measurement of the first ion momentum distributions Becker and Faisal made the first theoretical predictions [69] for double ionisation of He by evaluating the dominant Feynman diagram from the Intense-Field Many-Body S-Matrix Theory (c.f. Fig. 2b). The results for the momentum distributions of the $\mathrm{He}^{2+}$ ion calculated as the sum momentum of the two electrons are shown in Fig. 3(c,d) (solid squares). The double peak structure, its width and the position of the maxima for the parallel component (panel c) are reproduced by the calculations as well as the single hump structure for the perpendicular component (panel d). The minimum at momentum zero in the distribution for the parallel momentum is more pronounced in the calculation than in the data, which might be due to non-negligible contributions from higher-order diagrams (see also discussion below). To 


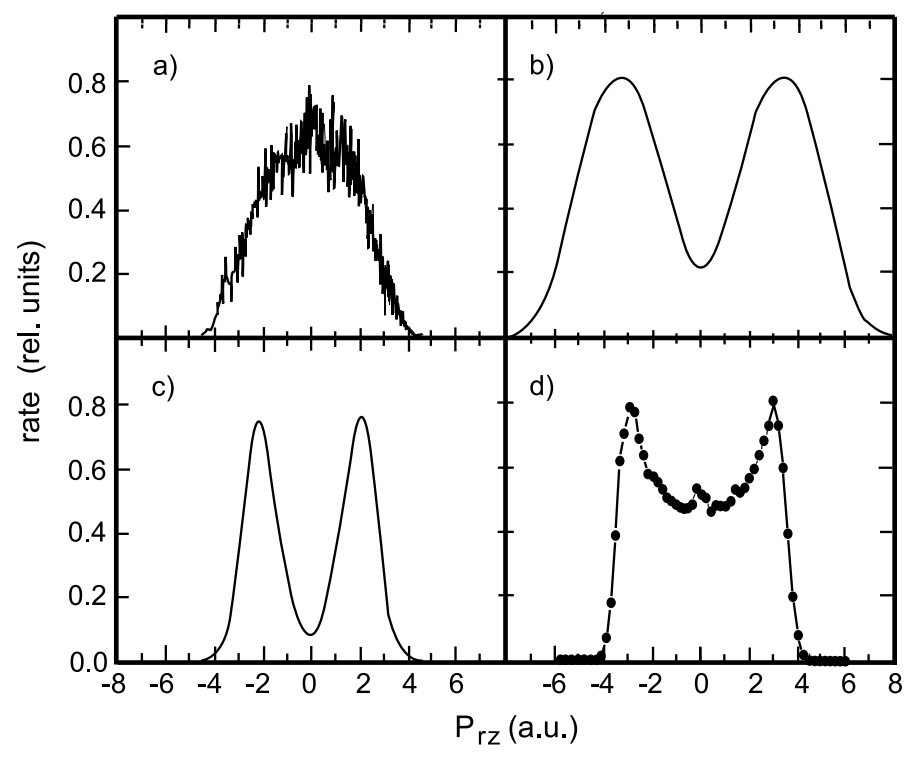

FIG. 4: Momentum distributions of $\mathrm{He}^{2+}$ ions at an intensity of $6.6 \times 10^{14} \mathrm{~W} / \mathrm{cm}^{2}$ for all panels. $p_{r z}$ is the component parallel to the laser polarisation. a) Solution of the one dimensional Schrödinger equation [76], b) Classical Trajectory Monte Carlo calculations [77], c) S-matrix calculation with additional saddle point approximation [71], and d) Wannier type calculation [74] .

unveil the physical origin responsible for the double hump structure Becker and Faisal have evaluated the rescattering diagram also by replacing the final Volkov (or field dressed) states by plane waves. Physically, this corresponds to switching off the laser field after both electrons are in the continuum. In the calculation this leads to a collapse of the double peak structure to a single peak (open squares in Fig. 3(c)), which confirms the interpretation given above, that it is the acceleration of the ion (or the electrons, respectively) in the field after the rescattering, which leads to the large ion momenta. The distribution perpendicular to field does not change (Fig. 3(d)), since the final state momenta of the electron do not couple to the field in this direction. It has been further shown by Jaroń and Becker [70] that the component of the sum-momentum parallel to the field is largest when the drift energy of the active electron in the intermediate state is close to zero.

The rescattering diagram has been also estimated by introducing different approximations in the evaluation. A stationary phase approximation has been used to evaluate the integrations over the time and the momentum in the intermediate state. This additional approximation reduces the computation time significantly and does not change the calcu- 
lated ion momentum distribution significantly as shown by Goreslavskii and Popruzhenko (Fig. 4c [71, 72]). Kopold et al. [73] further replaced the electron-electron interaction by a zero-range contact potential. Using these approximations they were to able to show that a (third-order) diagram, which implies an excitation of the $\mathrm{He}^{+}$ion via the electron-electron interaction, followed by the ionisation of the excited ion by the field, gives contributions near zero momentum of the recoil ion and may fill the valley in the parallel momentum distributions of the minimum at zero momentum.

A conceptionally different approach was used by Sacha and Eckhardt [74]. They argued that rescattering will produce a highly excited intermediate complex, which will then decay in the presence of the field. In a Wannier type analysis they studied the decay using classical trajectories in the saddle potential created by the field and the Coulomb potentials. Interestingly the recoil ion momentum exhibits a double peak structure, that does not depend strongly on the time of creation but on the energy. Sacha and Eckhardt find parallel and perpendicular momentum distributions, which are for helium (Fig. 4d) and neon in reasonable agreement with the experiment. Recently, they extended this model to examine the decay of highly excited three electron atoms [75].

Computation of the time dependent Schrödinger equation for two electrons in three dimensions is extremely challenging and there are no predictions of recoil ion momenta or other differential information in the "long" wavelength regime of presently available high-intensity lasers up to now. Lein et al. [76] reported the first results on recoil ion momenta based on an integration of the one-dimensional Schrödinger equation (see Fig. 4a). The momentum distribution peaks at zero momentum in contrast to all other results. This might be due to a well known problem of one-dimensional calculations, namely that the effect of electron repulsion is overemphasised.

Chen et al. [77] have performed a Classical Trajectory Monte Carlo calculation (CTMC) in which they solved the classical Hamilton equations of motion for all three particles in the field. The initial state in the simulation is determined by the momentum distributions following from tunneling of one electron from the atom. The results of this calculation also yields the observed double peak structure (Fig. 4b).

Nevertheless, the interpretation remains puzzling, if one compares recoil ion momentum distributions for different targets. In Fig. 5 experimental data for the parallel component as obtained by de Jesus et al. [59] for the doubly charged helium, neon and argon ions in 23 fs, 


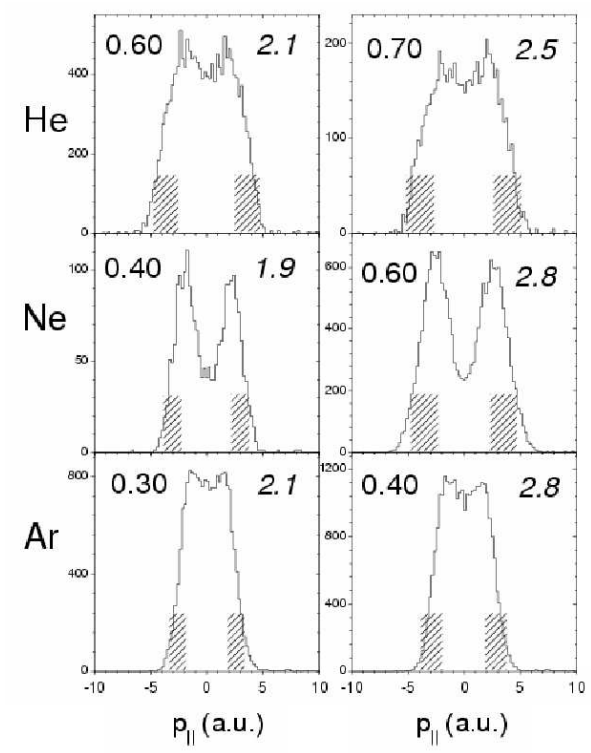

FIG. 5: Longitudinal ion momentum distributions for double ionisation of $\mathrm{He}, \mathrm{Ne}$ and $\mathrm{Ar}$ at intensities as indicated in the upper left corner of each panel (in units of $10^{15} \mathrm{~W} / \mathrm{cm}^{2}$ ). Ratios of the maximum recollision impact energy to the ionisation potential of the second electron, $E_{\text {rec }} / I_{p}$, are plotted in the upper right corners. The shaded areas represent the regions of the most probable momenta assuming classical electron motion and direct impact ionisation.

linearly polarised Ti:sapphire laser pulses at intensities between $0.35 \times 10^{15} \mathrm{~W} / \mathrm{cm}^{2}$ and $1.25 \times$ $10^{15} \mathrm{~W} / \mathrm{cm}^{2}$ are shown. While the pronounced double peak structure along the polarisation direction is found for the neon target at all intensities, this feature is considerably less apparent or even absent at the lowest intensity for the other two targets. Similar observations have been made for helium by Weber et al. [54] and for neon by Eremina et al. [64]. According to the semi-classical rescattering model the final drift momentum of the ion should depend on ratio of the maximum recollision energy, $E_{r e c}$, to the ionisation potential of the second electron, $I_{p}$, only. Note that the data in Fig. 5 are taken at similar ratios of $E_{r e c} / I_{p}$ (indicated in the upper left corner of each panel). Obviously, the distributions show a strong target dependence.

An explanation for this observation has been put forward by de Jesus et al. [59] based on the relative strength of the two mechanisms, namely the direct ionisation of the second electron via rescattering and its excitation via rescattering followed by subsequent field ionisation. In the latter mechanism ions with small momenta are generated. de Jesus et al. 

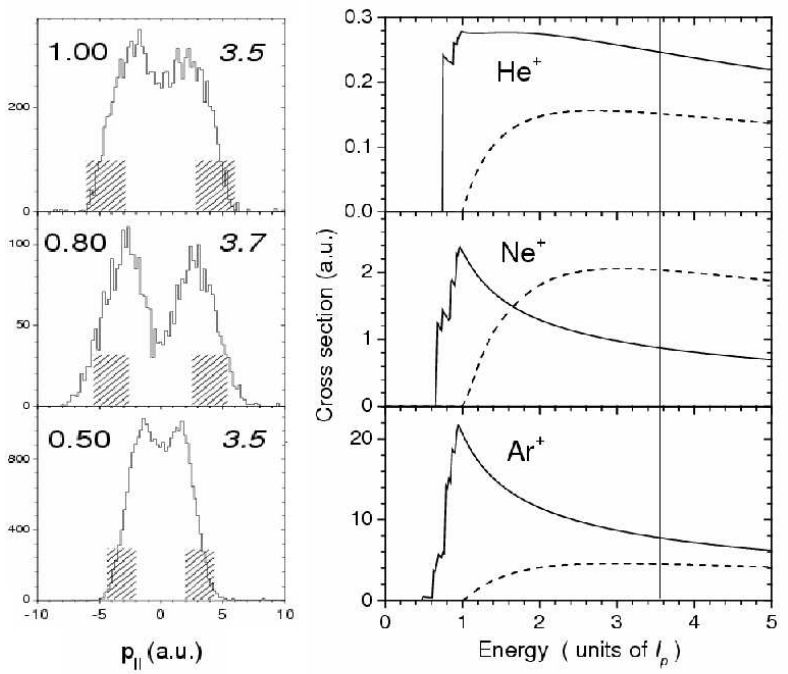

FIG. 6: Left panel: Ion momentum distributions as in fig.5 but for slightly higher intensities. Right panel: Total excitation (solid lines) and ionisation (dashed lines) cross sections of $\mathrm{He}^{+}, \mathrm{Ne}^{+}$and $\mathrm{Ar}^{+}$as a function of the electron impact energy in units of the ionisation potential. The vertical line indicates the maximum recollision energy for the present intensities.

have found that the contribution of the latter mechanism can exceed those of the former for He and Ar but not for Ne, which explains the differences between the measured recoil ion momentum distributions for the different targets (see Fig. 6). This is due to the fact that for He and Ar electron impact excitation cross sections for the singly charged ion exceed those for ionisation at all energies, which is not the case for Ne. It will be interesting to see in future, if calculations based on one of the theories discussed above can further substantiate these conclusions.

Momentum distributions of triply and fourfold charged ions have been observed by Moshammer et al. [55] for $\mathrm{Ne}^{3+}$ and by Rudenko et al. [60] up to $\mathrm{Ne}^{4+}$ and $\mathrm{Ar}^{4+}$. In Fig. 7 the results for the parallel momentum components observed in the latter experiment measured with 25 fs laser pulses at intensities of $1.2 \times 10^{15} \mathrm{~W} / \mathrm{cm}^{2}$ and $2 \times 10^{15} \mathrm{~W} / \mathrm{cm}^{2}$ are shown. The narrow distribution of the $\mathrm{Ne}^{2+}$ ions with a maximum at zero momentum indicates that at the present intensities double ionisation occurs via the sequential mechanism. At the same intensity the distributions for the higher charged states of Ne, however, exhibit a clear double peak structure with almost no ions created with zero momentum. 


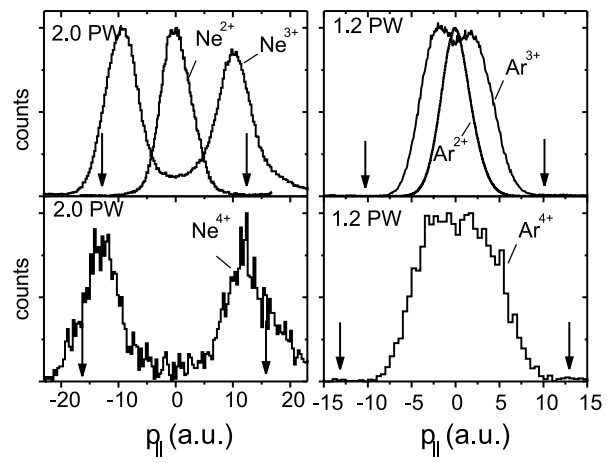

FIG. 7: Longitudinal momentum distributions of the recoil ion for double, triple and fourfold ionisation of $\mathrm{Ne}$ and $\mathrm{Ar}$ (see text).

The spectra extend slightly beyond $p_{\max }=2 n \sqrt{U_{p}}(n=3,4)$ (indicated by the arrows), which corresponds to the maximum classical longitudinal momentum the $\mathrm{Ne}^{n+}$ ion gains when the electrons are set free via a (e,ne)-rescattering event. For the multiply charged $\mathrm{Ar}$ ions the distributions are very different. The $\mathrm{Ar}^{3+}$ distributions exhibit a shallow minimum at zero, whereas for the $\mathrm{Ar}^{4+}$ ions there is no double peak structure at all. Further, both the distributions are much smaller than in the case of $\mathrm{Ne}$ and lie well within the classical limits of $2 n \sqrt{U_{p}}$ (see arrows). This indicates that for Ar at these intensities the direct (e,ne)-rescattering event is not the dominant mechanism.

\section{B. Electron energies}

Electron energy distributions for double ionisation have been reported for helium [49], argon [50, 52], neon [51] and xenon [48, 52]. A common feature of all these experiments is that the distributions generated via nonsequential double ionisation extend to much larger energies than those generated in single ionisation. Using the $S$-matrix theory Becker and Faisal have analysed [32] the data for He obtained by Lafon et al. [49] at $780 \mathrm{~nm}$. In Fig. 8 the results of calculations from the rescattering diagram (solid lines, c.f. Fig. 2b) and the shake-off diagram (dashed lines, c.f. Fig. 2a) are compared with the experimental data (circles) at (a) $4 \times 10^{14} \mathrm{~W} / \mathrm{cm}^{2}$ and (b) $8 \times 10^{14} \mathrm{~W} / \mathrm{cm}^{2}$. In both, experiment and calculation, the emission direction of one electron has been fixed along the polarisation direction while the energy and the emission direction of the other electron were kept unresolved (integrated 

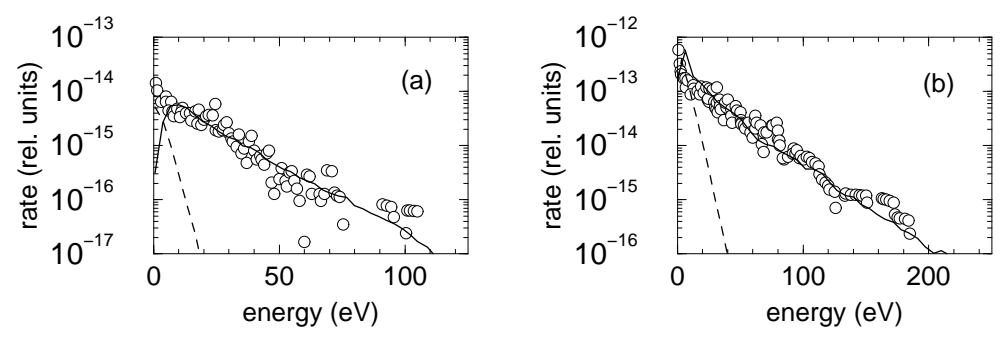

FIG. 8: Electron energy spectra from double ionisation of He at $780 \mathrm{~nm}$ and two intensities, (a) $4 \times 10^{14} \mathrm{Wcm}^{2}$ and (b) $8 \times 10^{14} \mathrm{~W} / \mathrm{cm}^{2} . S$-matrix results [32] from the rescattering diagram (solid lines) are compared with those from the shake-off diagram (dashed lines) and the experimental data (circles) obtained by Lafon et al. [49].

in theory). It is seen from the comparison that the experimental data are in full agreement with the theoretical predictions from the rescattering diagram, except for a narrow region near the threshold. In contrast, the results for the shake-off diagram decrease very quickly and fail completely to follow the trend of the experimental data.

Recently, Chaloupka et al. [52] have resolved distinct resonance-like structures in electron energy spectra of double ionisation of Xe, while in Ar featureless spectra, consistent with the rescattering mechanism, have been observed. They interpreted this observation as a progression from the tunneling and rescattering mechanism to a (unknown) multiphoton mechanism. There is no theoretical analysis of these observations up to now, and the underlying physics remains unclear.

\section{Correlated electron momenta}

More information can be obtained from the momentum correlation between the two electrons. In an experiment one possible choice would be to observe the momenta of both electrons in coincidence. In this case the recoil ion momentum could be calculated from the momentum conservation. From an experimental point of view however, it is easier to detect the ion and one of the electrons, in which case the momentum of the second electron can be inferred from momentum conservation. It is experimentally simpler, since the additional knowledge of the ion charge state, allows for an effective suppression of random 


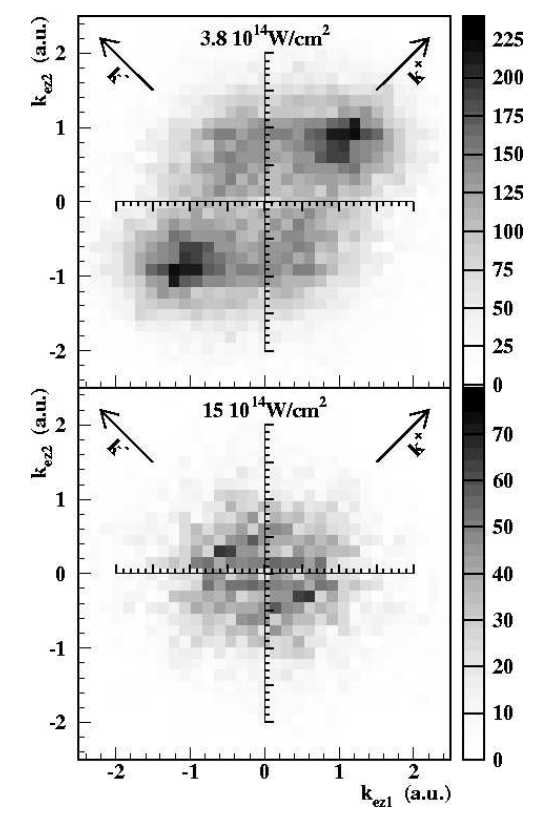

FIG. 9: Momentum correlation between the two emitted electrons when an $\mathrm{Ar}^{2+}$ ion is produced in the focus of a $220 \mathrm{fs}, 800 \mathrm{~nm}$ laser pulse at peak intensities of $3.8 \times 10^{14} \mathrm{~W} / \mathrm{cm}^{2}$ and $15 \times 10^{14} \mathrm{~W} / \mathrm{cm}^{2}$. The horizontal axis shows the momentum component of one electron along the polarisation of the laser field; the vertical axis represents the same momentum component of the corresponding second electron. Same sign of the momenta for both electrons represents an emission to the same half sphere. The data are integrated over the momentum components in the direction perpendicular to the polarisation direction. The gray shading shows the differential rate in arbitrary units on a linear scale (adapted from [61]).

coincidences. Moreover, electron and ion are detected on opposite detectors circumventing possible problems of multihit detection. Many successful studies for single photon double ionisation have been performed this way [34, 78-80].

Measurements of the momentum components parallel to the field of electron and ion while integrating over all the other momentum components have been reported by Weber et al. [61], Feuerstein et al. [63], Moshammer et al. [67] and de Jesus et al. [68]. The data of the first observations from double ionisation of Ar are shown in Fig. 9 [61]. Events in the first and third quadrant are those where both electrons are emitted to the same hemisphere, the second and fourth quadrant correspond to emission to opposite half spheres. The upper panel shows the electron momenta at an intensity of $3.6 \times 10^{14} \mathrm{~W} / \mathrm{cm}^{2}$, which is in the regime 


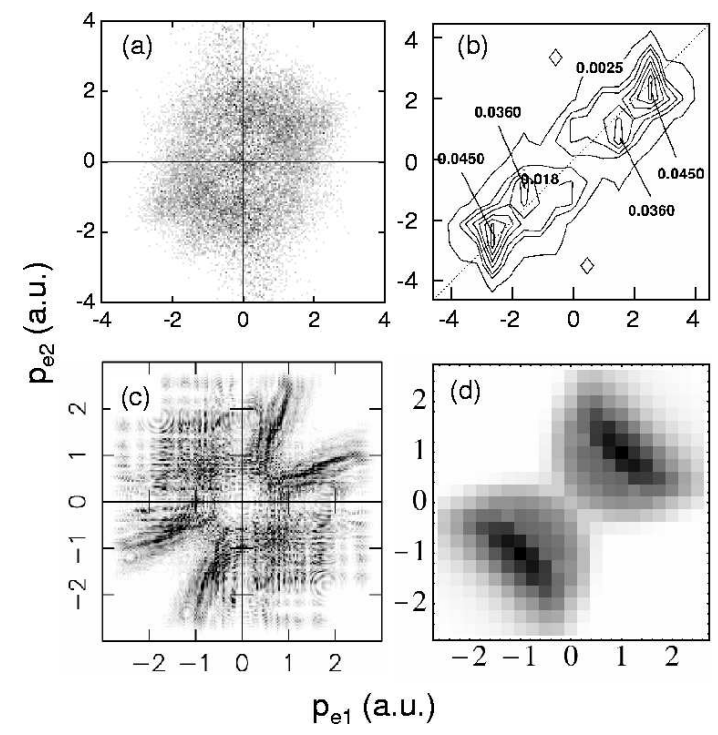

FIG. 10: Components of the correlated momenta of the electrons parallel for the field direction for double ionisation of He. (a) Experimental data at $5 \times 10^{14} \mathrm{~W} / \mathrm{cm}^{2}$ [68], (b) Results from the Classical Trajectory Monte Carlo calculation at $10^{15} \mathrm{~W} / \mathrm{cm}^{2}$ [77], (c) Results of one-dimensional numerical simulations at $7 \times 10^{14} \mathrm{~W} / \mathrm{cm}^{2}[76]$ and (d) results from $S$-matrix calculations at $6.6 \times 10^{14}$ $\mathrm{W} / \mathrm{cm}^{2}[32]$.

of nonsequential ionisation. The distribution shows a strong correlation between the two electrons, they are most likely emitted to the same hemisphere with a similar momentum of about 1 a.u.. At higher intensity, where double ionisation proceeds sequentially this correlation is lost (lower panel in figure 9).

In Fig. 10(a) the correlated momenta of the two electrons parallel to the polarisation direction for double ionisation of helium by $23 \mathrm{fs}, 5 \times 10^{14} \mathrm{~W} / \mathrm{cm}^{2}$, as measured by de Jesus et al. [68], are presented. Similar as for argon [61, 63, 64], but different from neon [67], a considerable amount of events appears in the second and fourth quadrant. Thus, the correlated momentum spectra indicate that as for Ar (c.f. [63]) also for He another mechanism, different from direct ionisation via rescattering, has to be present.

Also shown in Fig. 10 are theoretical predictions for the distribution of the electron momentum components parallel to the field direction at laser intensities close to the experimental one. First, the results from the Classical Trajectory Monte Carlo calculation (CTMC) by Chen et al [77] are shown in panel (b). Although excitation of the ion due 
to rescattering is included in their calculations, a tunneling ionisation of the (excited) ion is excluded. Accordingly, the distribution of the final electron momenta are found to be well within the classically allowed regime (the first and third quadrant) for direct ionisation by rescattering only. The results of a solution of the one-dimensional Schrödinger equation by Lein et al. [76] (Fig. 10c) show, in agreement with the experimental data, a considerable flux in the second and fourth quadrant. However, discrepancies to the experimental results are obtained along the diagonal in the first and third quadrant, when both electrons have the same longitudinal momentum. The maximum of the experimental distribution is located here with only a slight indication of a minimum along the diagonal, whereas a distinct minimum (zero) is found in the theory. This is obviously due to the restriction to an one-dimensional model, in which the electron-electron interaction is overestimated. Finally, panel (d) shows the results from the rescattering diagram of the $S$-matrix theory (c.f. Fig. 2b) obtained by Becker and Faisal [32]. Again, the distribution is overwhelmingly located in the first and third quadrant. Similar results have been obtained by other authors [81] calculating the rescattering diagram using additional approximations. This result underlines that the significant probabilities in the second and fourth quadrant go beyond the direct ionisation by recollision. It would be interesting to see if the prediction [63] that an excitation of the ion upon recollision followed by ionisation of the excited ion is responsible for these contributions can be substantiated by theoretical calculations. Experimentally this prediction has been supported by more complete experiments (see Fig. 13) which will be discussed below.

For double ionisation of Ar Weckenbrock et al. [62] and Moshammer et al. [82] measured in addition to the momentum parallel to the field also the transverse momentum of the detected electron. Both find that the longitudinal correlation pattern strongly depends on this transverse momentum (see Fig. 11). If one electron is emitted with any transverse momentum larger than 0.1 a.u. (i.e. with some angle to the polarisation axis) one mostly finds both electrons with a similar momentum component in the field direction. It is this configuration which dominates the integrated spectrum in Fig. 9. If, however, one electron is emitted almost parallel to the polarisation with a very small transverse momentum of $p_{\perp}<0.1$ a.u. one finds that the parallel momentum distribution does no longer peak on the diagonal. In this case most likely one electron is fast and the other slow. Recently, it has been shown by Figueira de Morisson Faria et al. [83, 84] that this is due to a strong 


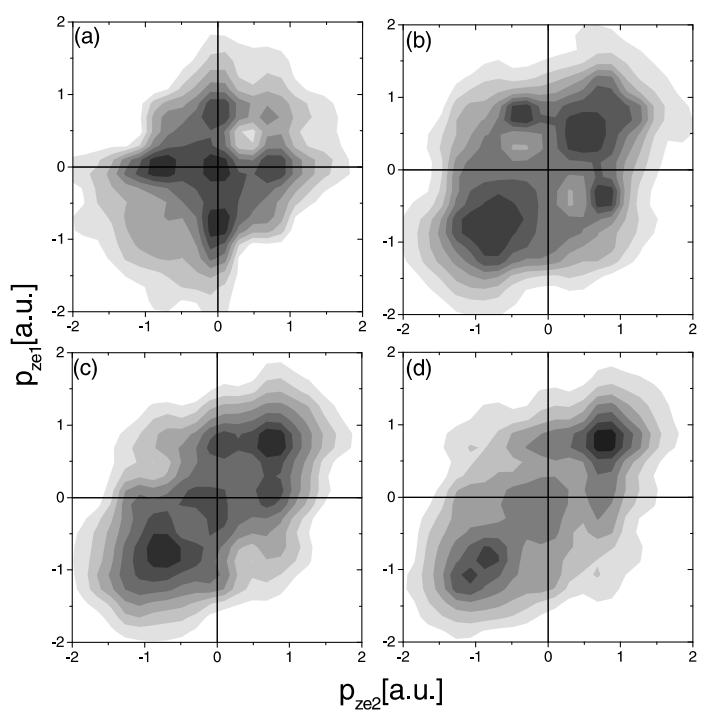

FIG. 11: Momentum correlation between the two emitted electrons when an $\mathrm{Ar}^{2+}$ ion is produced in the focus of a $150 \mathrm{fs}, 780 \mathrm{~nm}$ laser pulse at peak intensities of $4.7 \times 10^{14} \mathrm{~W} / \mathrm{cm}^{2}$. Axis as in Fig. 9. Each panel represents a part of the final state for a fixed transverse momentum $\left(p_{\perp}\right)$ of one of the electrons. (a) One of the electrons has a transverse momentum of $p_{\perp}<0.1$ a.u., (b) $0.1<p_{\perp}<0.2$ a.u., (c) $0.2<p_{\perp}<0.3$ a.u., (d) $0.3<p_{\perp}<0.4$ a.u.. The gray scale shows the differential rate in arbitrary units and linear scale (from [62]).

electron-electron interaction between the two electrons in the final state.

Recently, Weckenbrock et al. [65] investigated in a joint experimental and theoretical study the momentum balance in the direction perpendicular to the polarisation axis. In this kinematical geometry the subtleties of the Coulombic interaction in the rescattering processes can be tested, since the perpendicular momentum components are not affected by the field. In Fig. 12 the distribution of the momentum components perpendicular to the polarization axis of one of the electrons, $b$, is plotted. The momentum component of the other electron in the same plane is shown by the arrow, the parallel components of both momenta as well as the magnitude of the momentum of electron $a$ are not resolved in the experiment (integrated in the theory). The experimental data show clearly, that the two electrons are emitted to opposite sides. This back-to-back emission is found to be due to a strong interaction between the two electrons after their double escape, as can be seen from the results of $S$-matrix calculations displayed in the other panels. Theoretical predictions and experimental data are in good agreement with each other (Fig. 12), when 


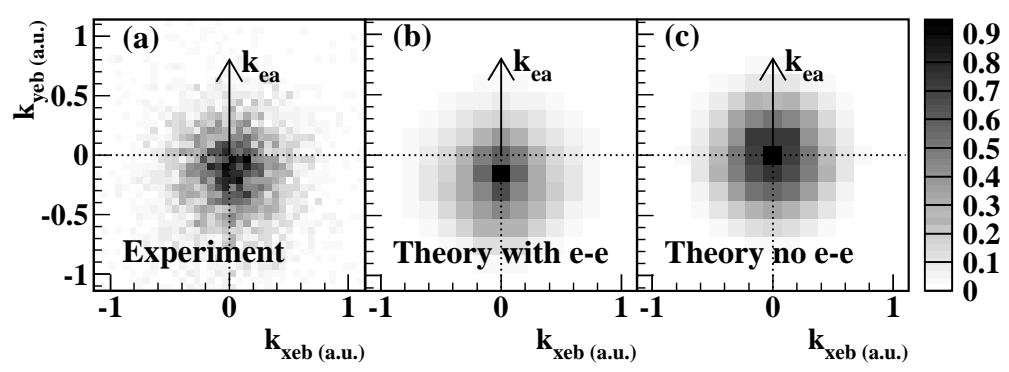

FIG. 12: Momentum distribution of electron $b$ in the plane perpendicular to the field direction for the double ionisation of Ar at $780 \mathrm{~nm}$ and $1.9 \times 10^{14} \mathrm{~W} / \mathrm{cm}^{2}$ [65]. The perpendicular momentum direction of the other electron, $a$, is shown by the arrow. The data are integrated over all momentum components along the polarisation direction and the magnitude of the momentum of electron $a$. A comparison is shown between (a) the experimental data, (b) theoretical results from the extended rescattering diagram including electron-electron interaction in the final state and (c) theoretical result from the rescattering diagram without electron-electron interaction in the final state.

the rescattering diagram (Fig. 2b) is extended such that the full electron-electron interaction is taken into account in the final state (panel b). The results neglecting the electron-electron interaction (as in the original rescattering diagram, Fig. 2b) do not show the back-to-back characteristics (Fig. 12). This reveals the importance of the final-state repulsion between the electrons for the nonsequential double ionisation process. In the experiment a back-to-back correlation between one of the electrons and the ion has been observed too. This could not be reproduced by the predictions from rescattering diagrams, which do not include effects of the electron-ion interaction and the collisional excitation process.

Kinematically complete experiments, i.e. experiments in which all momentum components of all particles have been reported by Weckenbrock et al. [85] for double ionisation of neon when the recollision energy of the first electron is on the order of the ionisation potential. The experiment finds, that the electron repulsion in the perpendicular plane (as for example in Fig. 12) is only present if both electrons have similar longitudinal momenta (Fig. 13). In contrast, repulsion is not visible for events where the two electrons are located in the second and fourth quadrant (Fig. 13a), i.e. are driven by the field in opposite direction. These findings directly supports the interpretation of parallel momenta as time stamps which we put forward throughout this review: Correlated electron momentum detection is a 

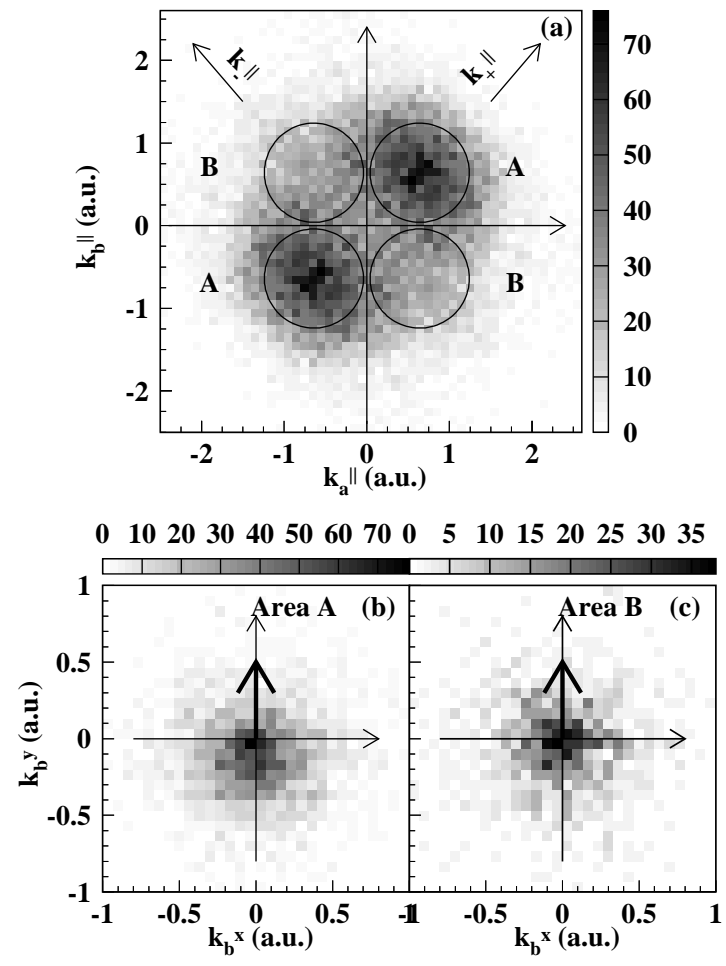

FIG. 13: Double ionisation of Neon at $1.9 \times 10^{14} \mathrm{~W} / \mathrm{cm}^{2}, 800 \mathrm{~nm}, 40$ fsec. (a) horizontal axis: momentum of electron a parallel to the polarisation direction, vertical axis: momentum of electron $\mathrm{b}$ parallel to the polarisation direction. The areas indicated by the circles show the region of events selected in panel b) and c). (b) Momentum components of electron b in the plane perpendicular to the polarisation, the perpendicular momentum of electron a is along the positive y axis as shown by the arrow. Only events within the circles in region A have been selected. (c) same as (b) but for events in circles in region B (from [85])

subcycle time correlation measurement on the attosecond time scale. For the case that the recolliding electron is close to the ionisation threshold, which is well fulfilled in experiment shown in Fig. 13, the difference in the parallel momenta between the electrons correspond to the difference of their time of birth. Electrons in regions A (Fig. 13a) are both created at the time of recollision while electrons in region B are emitted at different times. In consequence, electrons in region A show repulsions in the perpendicular plane while those in region $\mathrm{B}$ do not feel their repulsion. The latter electrons are likely produced via recollision with excitation followed by delayed field ionisation as discussed above.

The final step towards complete information on the strong field double ionisation process 


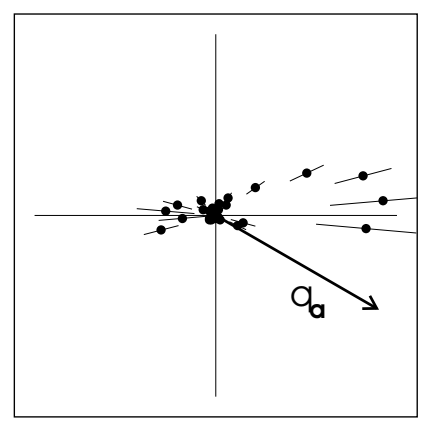

FIG. 14: Double ionisation of Neon at $1.9 \times 10^{14} \mathrm{~W} / \mathrm{cm}^{2}, 800 \mathrm{~nm}, 40$ fsec. Angular distribution of electron b. The polarisation axis is horizontal, the sum energy of electron a and b is $10 \mathrm{eV}<$ $\left(E_{a}+E_{b}\right)<24 \mathrm{eV}$. Both electrons have the same energy (from [85])

is to investigate the angular distribution of one electron of well defined energy for fixed angle and energy of the second electron. First successful steps towards this goal have been done for the multiphoton case as well [85]. Fig. 14 shows one of those angular distributions. They highlight that most of the electrons are emitted with only a small angle between them with some influence of the electron repulsion still visible. There is however a smaller second lobe of electrons which are emitted back to back. Once such studies are performed with an energy resolution on the electron sum energy below the single photon energy, it can be expected that dipole selection rules become visible in these distributions as they dominate the structure of the three-particle continuum for the single photon case.

\section{OUTLOOK}

In the future, we will certainly experience a continuation of the rapid progress on either sides, the COLTRIMS technique and in laser technology, and theoretical efforts towards abinitio simulations and many-electron emission. For the laser technology, efforts concentrate to produce ultrashort pulses with only two or three optical cycles as well as to control the phase of the carrier-wave within the pulse envelope over reasonably long time-scales. With such pulses it will be possible not only to precisely adjust the temporal shape of the electric field of the light wave but also to control its direction and strength at the instant of electron recollision. It can be anticipated that this will enable new insights into the many aspects of the electron dynamics in double ionisation on fs or even sub-fs time scales. Pioneering 
measurements for single ionisation indicated already a strong dependence of the electron emission characteristics on the carrier-envelope phase. On the experimental side, though kinematically complete data sets on double ionisation of Ne have been collected for the first time, further experiments with improved statistics are certainly needed to ultimately test theory and to disentangle the still unexplained target dependences. Clearly, most desirable are fully differential data with good resolution for double ionisation of He, the prototype system for theory. With the advent of larger computer systems one can anticipate that abinitio simulations in this simplest two-electron system interacting with infrared laser pulses will become available in the next years. This will further help in unveiling the temporal details of the mechanism leading to nonsequential double ionisation and may lead also to improved models for multiple ionisation. Concerning triple or even quadruple ionisation, so far only the ion momenta have been measured for Ar and Ne. In order to obtain conclusive answers about the involved reaction pathways as well as on their dependences on the target structure measurements differential in the electron momenta represent most likely the next step.

Another important future direction are studies of the wavelength dependence of double ionisation. At present, experimental data are at hand only for the two extreme cases, namely single and multiphoton absorption (more than 50 photons for the case of He). The intermediate regime of two or three photon two-electron transitions is experimentally completely unexplored. This will change in the very near future when the free-electron laser at the TESLA Test facility in Hamburg will start its operation in the beginning of 2005 . It will open exciting possibilities to explore a new regime of non-linear multi-photon processes, where only a few high-energy photons interact with few electrons. Experiments for twophoton double ionisation of helium are under preparation. Theoretical investigations in this direction have already begun.

\section{Acknowledgements}

A.B. acknowledges support from the Alexander von Humboldt-Stiftung and from the Deutsche Forschungsgemeinschaft. He also would like to acknowledge F.H.M. Faisal for the joint work on the IMST and its application to strong-field double ionization and indispensable discussions on this topic over many years, he also thanks A. Jaron-Becker for 
her contributions and many discussions. R.D. particularily thanks Th. Weber, H. Giessen, M. Weckenbrock, A. Staudte, P.B. Corkum, D. Zeidler and M. Smolarski for their contributions to the experiments and their interpretation. He acknowledges financial support by DFG and BMBF. R.M. acknowledges support from the Max-Planck-Society and from the Deutsche Forschungsgemeinschaft within the Leibniz programme. He would like to thank A. Rudenko, B. Feuerstein, C.D. Schröter, K. Zrost and J. Ullrich, who decisively contributed in performing the experiments as well as in evaluation and interpretation of the data. We are indebted to thank for numerous discussions our colleagues and friends W. Becker, S.L. Chin, E. Eremina, D. Dundas, M. Lein, H. Reiss, J.M. Rost, H. Rottke, W. Sandner, K. Taylor and many others.

[1] A. Einstein, Ann. d. Phys. 17, 132 (1905).

[2] M. Göppert-Mayer, Ann. d. Phys. 9, 273 (1931).

[3] The atomic unit of electric field strength felt by an electron on the first Bohr orbit of hydrogen atom is $\mathcal{E}_{a}=e / a_{0}^{2} \simeq 5.1 \times 10^{9} \mathrm{~V} / \mathrm{cm}$, the corresponding intensity being $I_{a}=3.51 \times 10^{16}$ $\mathrm{W} / \mathrm{cm}^{2}$. It corresponds to about $10^{10}$ coherent photons in a box of the size of the wavelength of a Ti:sapphire laser $(800 \mathrm{~nm})$.

[4] K. Burnett, V. Reed, and P. Knight, J. Phys. B 26, 561 (1993).

[5] L. DiMauro and P. Agostini, Adv. At. Mol. Opt. Phys. (Academic Press, New York, 1995),

[6] M. Protopapas, C. Keitel, and P. Knight, Rep. Prog. Phys. 60, 389 (1997).

[7] F. Ehlotzky, A. Jaroń, and J. Kamiński, Phys. Rep. 297, 63 (1998).

[8] C.J. Joachain, M. Dörr, and N.J. Kylstra, Adv. At. Mol. Opt. Phys. 42, 225 (2000).

[9] F. Ehlotzky, Phys. Rep. 345, 175 (2001).

[10] W. Becker, F. Grasbon, R. Kopold, D.B. Milosevic, G.G. Paulus, and H. Walther, Adv. At. Mol. Opt. Phys. 48, 35 (2002).

[11] D.B. Milosevic, and F. Ehlotzky, Adv. At. Mol. Opt. Phys. 49, 373 (2003).

[12] J. McGuire, Electron Correlation Dynamics in Atomic Collisions (Cambridge, Cambridge University Press, 1997).

[13] J. Briggs and V. Schmidt, J. Phys. B 33, R1 (2000).

[14] R. Doerner, H. Schmidt-Böcking, Th. Weber, T. Jahnke, M. Schöffler, A. Knapp, M. Hattass, 
A. Czasch, L.Ph.H. Schmidt, and O. Jagutzki, Rad. Phys. Chem. 70, 191 (2003).

[15] J. Parker, K.T. Taylor, C.W. Clark, and S. Blodgett-Ford, J. Phys. B 29, L33 (1996).

[16] D. Dundas, K. Taylor, J. Parker, and E. Smyth, J. Phys. B 32, L231 (1999).

[17] K. Taylor, J. Parker, D. Dundas, E. Smyth, and S. Vitirito, Laser Phys. $\underline{\mathbf{9}}, 98$ (1999).

[18] J. S. Parker, L. R. Moore, D. Dundas, and K. T. Taylor, J. Phys. B 33, L691 (2000).

[19] J. S. Parker, L. R. Moore, K. J. Meharg, D. Dundas, and K. T. Taylor, J. Phys. B 34, L69 (2001).

[20] J. Parker, B.J.S. Doherty, K.J. Meharg, and K.T. Taylor, J. Phys. B 36, L393 (2003).

[21] K.C. Kulander, Phys. Rev. A 38, 778 (1988).

[22] B. Walker, B. Sheehy, L.F. DiMauro, P. Agostini, K.J. Schafer, and K.C. Kulander, Phys. Rev. Lett. 73, 1227 (1994).

[23] D. Charalambidis, D. Xenakis, C.J.G.J. Uiterwaal, J. Zhang, H. Schröder, O. Faucher, and P. Lambropoulos, J. Phys. B 30, 1467 (1997).

[24] S. Larochelle, A. Talebpour, and S.L. Chin, J. Phys. B 31, 1215 (1998).

[25] A. Becker, and F.H.M. Faisal, Phys. Rev. A 59, R1742 (1999).

[26] A. Becker, and F.H.M. Faisal, Phys. Rev. A 59, R3182 (1999).

[27] A. L'Huillier, L.A. Lompre, G. Mainfray, and C. Manus, Phys. Rev. Lett. 48, 1814 (1982).

[28] K. Hino, T. Ishihara, F. Shimizu, N. Toshima, and J.H. McGuire, Phys. Rev. A 48, 1271 (1993).

[29] A. Kheifets, I. Bray, K. Soejima, A. Danjo, K. Okuno, and A. Yagishita, J. Phys. B 34, L247 (2001).

[30] F.H.M. Faisal, and A. Becker, in Selected Topics on Electron Physics (Plenum Press, New York, 1996) 397.

[31] A. Becker, and F.H.M. Faisal, J. Phys. B 29, L197 (1996).

[32] A. Becker, and F.H.M. Faisal, Phys. Rev. Lett. 89, 193003 (2002).

[33] A. Becker, and F.H.M. Faisal, J. Phys. B (Topical Review, in press).

[34] R. Dörner, H. Bräuning, J. Feagin, V. Mergel, O. Jagutzki, L. Spielberger, T. Vogt, H. Khemliche, M. Prior, J. Ullrich, Phys. Rev. A 57, 1074 (1998).

[35] D.N. Fittinghoff, P.R. Bolton, B. Chang, and K.C. Kulander, Phys. Rev. Lett. 69, 2642 (1992).

[36] M. Y. Kuchiev, Sov. Phys. JETP Lett. 45, 404 (1987).

[37] P. Corkum, Phys. Rev. Lett. 71, 1994 (1993). 
[38] K. Schafer, B. Yang, L. DiMauro, and K. Kulander, Phys. Rev. Lett. 70, 1599 (1993).

[39] J. Samson, Phys. Rev. Lett. 65, 2863 (1990).

[40] U. Eichmann, M. Dörr, H. Maeda, W. Becker, and W. Sandner, Phys. Rev. Lett. 84, 3550 (2000).

[41] L.V. Keldysh, Sov. Phys. JETP 20, 1307 (1965).

[42] D. Fittinghoff, P. Bolton, B. Chang, and K. Kulander, Phys. Rev. A 49, 2174 (1994).

[43] P. Dietrich, N. H. Burnett, M. Ivanov, and P. B. Corkum, Phys. Rev. A 50, R3585 (1994).

[44] B. Zon, Sov. Phys. JETP 89, 219 (1999).

[45] A. Becker, and F.H.M. Faisal, J. Phys. B 32, L335 (1999).

[46] A. Becker, F.H.M. Faisal, Y. Liang, S. Augst, Y. Beaudoin, M. Chaker, and S.L. Chin, J. Phys. B 33, L547 (2000).

[47] H. Maeda, M. Dammasch, U. Eichmann, W. Sandner, A. Becker, and F.H.M. Faisal, Phys. Rev. A 62, 035402 (2000).

[48] B. Witzel, N. A. Papadogiannis, and D. Charalambidis, Phys. Rev. Lett. 85, 2268 (2000).

[49] R. Lafon, J. L. Chaloupka, B. Sheehy, P. M. Paul, P. Agostini, K.C. Kulander, and L. F. DiMauro, Phys. Rev. Lett. 86, 2762 (2001).

[50] E. Peterson and P. Bucksbaum, Phys. Rev. A 64, 053405 (2001).

[51] R. Moshammer, B. Feuerstein, D. Fischer, A. Dorn, C. Schröter, J. Deipenwisch, J. LopezUrrutia, C. Höhr, P. Neumayer, J. Ullrich, Optics Express $\underline{\mathbf{8}}, 358$ (2001).

[52] J. L. Chaloupka, J. Rudati, R. Lafon, P. Agostini, K.C. Kulander, and L. F. DiMauro, Phys. Rev. Lett. 90, 033002 (2003).

[53] J. Ullrich, R. Moshammer, A. Dorn, R. Dörner, L.Ph. Schmidt, and H. Schmidt-Böcking, Rep. Prog. Phys. 66, 1463 (2003).

[54] T. Weber, M. Weckenbrock, A. Staudte, L. Spielberger, O. Jagutzki, V. Mergel, G. Urbasch, M. Vollmer, H. Giessen, and R. Dörner, Phys. Rev. Lett. 84, 443 (2000).

[55] R. Moshammer, B. Feuerstein, W. Schmitt, A. Dorn, C. Schröter, J. Ullrich, H. Rottke, C. Trump, M. Wittmann, G. Korn, Phys. Rev. Lett. 84, 447 (2000).

[56] T. Weber, M. Weckenbrock, A. Staudte, L. Spielberger, O. Jagutzki, V. Mergel, G. Urbasch, M. Vollmer, H. Giessen, and R. Dörner, J. Phys. B 33, L127 (2000).

[57] B. Feuerstein, R. Moshammer, and J. Ullrich, J. Phys. B 33, L823 (2000).

[58] T. Weber, O. Jagutzki, M. Hattass, A. Staudte, A. Nauert, L. Schmidt, M. Prior, A. Landers, 
A. Bräuning-Demian, H. Bräuning, J. Phys. B 34, 3669 (2001).

[59] V.L.B. deJesus, B. Feuerstein, K. Zrost, D. Fischer, A. Rudenko, F. Afaneh, C.D. Schröter, R. Moshammer, and J.. Ullrich, J. Phys. B 37, L161 (2004).

[60] A. Rudenko, K. Zrost, C.D. Schröter, V.L.B. deJesus, B. Feuerstein, R. Moshammer,and J. Ullrich, Phys. Rev. Lett. (accepted for publication) (2004).

[61] T. Weber, H. Giessen, M. Weckenbrock, A. Staudte, L. Spielberger, O. Jagutzki, V. Mergel, G. Urbasch, M. Vollmer, and R. Dörner, Nature 404, 608 (2000).

[62] M. Weckenbrock, M. Hattass, A. Czasch, O. Jagutzki, L. Schmidt, T. Weber, H. Roskos, T. Löffler, M. Thomson, and R. Dörner, J. Phys. B 34, L449 (2001).

[63] B. Feuerstein, R. Moshammer, D. Fischer, A. Dorn, C. D. Schröter, J. Deipenwisch, J. LopezUrrutia, C. Höhr, P. Neumayer, J. Ullrich, Phys. Rev. Lett. 87, 043003 (2001).

[64] E. Eremina, X. Liu, H. Rottke, W. Sandner, A. Dreischuh, F. Lindner, F. Grasbon, G.G. Paulus, H. Walther, R. Moshammer, B. Feuerstein, and J. Ullrich, J. Phys. B 36, 3269 (2003).

[65] M. Weckenbrock, A. Becker, A. Staudte, S. Kammer, M. Smolarski, V.R. Bhardwaj, D.M. Rajner, D.M. Villeneuve, P.B Corkum, and R. Dörner, Phys. Rev. Lett. 91, 123004 (2003).

[66] M. Weckenbrock, D. Zeidler, A. Staudte, Th. Weber, M. Schöffler, M. Meckel, S. Kammer, M. Smolarski, O. Jagutzki, V.R. Bhardwaj, D.M. Rajner, D.M. Villeneuve, P.B Corkum, and R. Dörner, Phys. Rev. Lett. 91, 123004 (2003).

[67] R. Moshammer, J.. Ullrich, B. Feuerstein, D. Fischer, A. Dorn, C.D. Schröter, J.R. Crespo López-Urrutia, C. Höhr, H. Rottke, C. Trump, M. Wittmann, G. Korn, K. Hoffmann,and W. Sandner, J. Phys. B (36), L113 (2003).

[68] V.L.B. deJesus, A. Rudenko, B. Feuerstein, K. Zrost, C.D. Schröter, R. Moshammer,and J.. Ullrich, J. Electr. Spectr. 141, 127 (2004).

[69] A. Becker and F.H.M. Faisal, Phys. Rev. Lett. 84, 3546 (2000).

[70] A. Jaroń and A. Becker, Phys. Rev. A. 67, 035401 (2003).

[71] S. Goreslavskii and S. Popruzhenko, Optics Express $\underline{\mathbf{8}}, 395$ (2001).

[72] S. Goreslavskii and S. Popruzhenko, J. Phys. B 34, L239 (2001).

[73] R. Kopold, W. Becker, H. Rottke, and W. Sandner, Phys. Rev. Lett. 푸, 3781 (2000).

[74] K. Sacha and B. Eckhardt, Phys. Rev. A 63, 043414 (2001).

[75] K. Sacha and B. Eckhardt, Phys. Rev. A 64, 053401 (2001). 
[76] M. Lein, E. Gross, and V. Engel, Phys. Rev. Lett. $\underline{\mathbf{8 5}}, 4707$ (2000).

[77] J. Chen, J. Liu, L. Fu, and W. Zheng, Phys. Rev. A 63, 011404(R) (2000).

[78] R. Dörner, J. Feagin, C. Cocke, H. Bräuning, O. Jagutzki, M. Jung, E. Kanter, H. Khemliche, S. Kravis, V. Mergel, Phys. Rev. Lett. 77, 1024 (1996), (see also erratum in Phys. Rev. Lett. 78, $2031(1997))$.

[79] V.Mergel, M. Achler, R. Dörner, K. Khayyat, T. Kambara, Y. Awaya, V. Zoran, B. Nyström, L.Spielberger, J. McGuire, Phys. Rev. Lett. 80, 5301 (1998).

[80] M. Achler, V. Mergel, L. Spielberger, Y. A. R. Dörner, and H. Schmidt-Böcking, J. Phys. B 34, L965 (2001).

[81] S. Goreslavskii, S. Popruzhenko, R. Kopold, and W. Becker, Phys. Rev. A 64, 053402 (2001).

[82] R. Moshammer, B. Feuerstein, J. Crespo Lopez-Urrutia, J Deipenwisch, A. Dorn, D. Fischer, C. Höhr, P. Neumayer, C.D. Schröter, J. Ullrich, H. Rottke, C. Trump, M. Wittmann, G. Korn, and W. Sandner, Phys. Rev. A 035401 (2002).

[83] C. Figueria de Morisson Faria, X. Liu, W. Becker, and H. Schomerus, Phys. Rev. A 69, 021402(R) (2004).

[84] C. Figueria de Morisson Faria, H. Schomerus, X. Liu, and W. Becker, Phys. Rev. A 69, 043405 (2004).

[85] M. Weckenbrock, D. Zeidler, A. Staudte, Th. Weber, M. Schöffler, M. Meckel, S. Kammer, M. Smolarski, O. Jagutzki, V.R. Bhardwaj, D.M. Rayner, D.M. Villeneuve, P.B. Corkum, and R. Dörner, Phys. Rev. Lett. 92, 213002 (2004). 